\title{
Clinical profile and early outcome of arteriovenous fistula creation for haemodialysis: Integrated activity in a general surgery unit
}

\author{
Deep Lamichhane, Sanjay Paudyal, Pukar Maskey, Santa Bir Maharjan, Arbin Joshi, Bimal Pandey, \\ Jay Narayan Shah, Vijay Kumar Jaiswal
}

Department of Surgery, Department of Surgery, Patan Hospital, Lalitpur, Nepal

Correspondence: Dr Deep Lamichhane, Patan Hospital, Lagankhel, Nepal.

Email: deeplamichhane@gmail.com

\begin{abstract}
Introduction: A functioning arteriovenous fistula (AVF) is essential for maintenance hemodialysis (HD) in a patient with chronic renal failure. This study explores the creation of AVF as an integral activity in a general surgical unit by a general surgeon.

Methods: This is a hospital based cross sectional study done at Patan Hospital patients who underwent creation of AVF from January 2015 to June 2015 were included in the study. Patient demographic and clinical data were documented. Outcome and complications of AVF surgery were obtained over a 6 months period from the time of vascular access creation.

Results: Thirty-seven patients were operated in the study period with a mean age of 35.44 years and $M$ : F ratio of 2.7:1. There were 3 immediate and 3 delayed failure with $81.81 \%(n=27)$ patency rate at 6 months follow up. The maturation time was 3-7weeks (mean +/- SD of $4.83+/-1.053$ ). Complication occurred in 4 patients, namely arm edema $(n=3)$ and haematoma $(n=1)$.

Conclusion: The creation of AVF for HD performed in a general surgery unit is a safe procedure with satisfactory outcome and acceptable level of complications.
\end{abstract}

Keywords: Arteriovenous fistula; chronic renal failure; general surgery unit; maintenance haemodialysis

\section{Introduction}

End stage renal disease (ESRD) is increasing worldwide and one of the treatment options for ESRD is maintenance hemodialysis (HD) through an arteriovenous fistula (AVF) until renal transplantation; therefore it is essential that AVF has a long term patency. ${ }^{1,2}$ AVFs are the preferred option for vascular access in patients requiring HD as they have relatively low rates of thrombosis, require fewer interventions to maintain patency, have a longer overall survival and have ability to withstand multiple needle puncture. $^{3}$ They are associated with reduced mortality compared with grafts or central venous catheters. ${ }^{4}$

Our hospital has a dedicated dialysis unit with $10 \mathrm{HD}$ machine running at full capacity so we get frequent referral for creation of AVF. Majority of patients with ESRD are treated with HD for life long as renal transplantation is not readily available in our set up. Given the high post operative failure rates and limited AVF sites, it has been proposed that AVF should be performed by vascular surgeon or in a vascular unit; both are not readily available in our rural parts of the country. However some studies claim good outcome can be obtained even if AVF is created by a surgical trainee or a general surgeon. ${ }^{5}$ The patient keeps on hopping and suffers a lot. The two main reasons besides others for failure of AVF are surgeon factors and improperly selected vessels. ${ }^{6}$

We sought to access outcome and complications of AVF surgery in a general surgical unit by a general surgeon and clinical profile of those patients. 


\section{Methods}

This is the hospital based cross-sectional study done from January to June 2015 in a general surgery unit of a medical college in Kathmandu. Thirty seven consecutive patients with ESRD requiring HD who were referred to surgical clinic for permanent vascular access were recruited. Vascular assessment was done by Allen's test, evaluation for venous thrombosis from previous cannulation and venous size was performed clinically by palpation. An elastic band was tied below elbow to distend the vein in distal forearm for assessment of venous system by palpation. All fistulas were Radio cephalic (Brescio-Cimino) made in a non dominant arm. We excluded patients who had thrombosed cephalic veins in distal forearm, patients with vein that was not deemed suitable for fistula because of small diameter or tortuous course, patients who were not co operative due to uremic symptoms and patients with negative Allen's test.

All surgeries were performed under Local anaesthesia (Lignocaine hydrochloride 1\%) on OPD basis by a single surgeon, who is interested in the subject but without working exclusively on vascular surgery (activity is that of any general surgeon). Semi lunar incision was made at level of distal forearm at the site of most suitable vein with convexity distally, cephalic vein was isolated, tied distally, cut and proximal portion was distended using normal saline with clamp proximally. The radial artery was isolated and cut longitudinally. End to side anastomosis was done using (continuous) Prolene 6-0 without using magnifying loop. Heparin was used to flush the vessels during anastomosis. Mechanical dilatation and vasodilator drugs were not used during the procedure. Immediate assessment of flow for the thrill, pulsation and distension of vein was noted and skin closed with staplers. All patients were kept for some time in recovery room and sent home with tramadol and ciprofloxacin. Patients were advised for exercise using soft ball from first post operative day. Follow up was done for palpation for thrill and auscultation for a bruit at day 1, 10 day, 4 weeks, 6 weeks and till 6 months. Fistulas with good thrills/ pulsation that were considered clinically mature were subjected to needling for HD and maturation time was noted. The study endpoints were vascular access patency at 6 months or failure. Patency was defined as "used for dialysis" if the AVF can be used for dialysis at the time of examination. The fistula complication such as hematoma was noted. The activity was carried out within the overall functioning of the department of general surgery without vascular surgeon involvement.

\section{Definitions}

Fistula was termed as successful if there is distension of vein or presence of thrill at the end of the procedure. Immediate failure is labeled when there is absent flow or thrill upto Day 10 follow up or if it was never used for dialysis. Delayed failure was labeled when it cannot be used for dialysis or there was no thrill at 6months follow up. Maturation time is when the fistula was used successfully for HD for the first time.

Ethical approval was taken from local ethical review board. Microsoft office excel 2013 package was used for data analysis. Continuous variables were presented as means +/- standard deviations.

\section{Results}

Thirty seven AVFs were created in the study period (Table 1). The mean age of patients was 35.44 years (14-76) with a male female ratio of 2.7:1. The most common cause of renal failure among the cause identified was chronic glomerulonephritis. All the patients were newly enrolled ESRD patients with maintenance HD except one in whom it was created before starting HD.

\section{Table 1: Demographic Profile of Patients who underwent surgery for AVF}

\begin{tabular}{ll} 
Causes of Renal Failure & \\
\hline Diabetes Mellitus & 2 \\
Hypertension & 2 \\
Polycystic Kidney Disease & 1 \\
Chronic Glomerulonephritis & 9 \\
Unidentified & 17 \\
Hypertension/ Diabetes & 3 \\
Obstructive Uropathy & 3
\end{tabular}

Brescio-Cimino fistula was created at left forearm in all patients. In one patient procedure was abandoned due to inability to isolate the cephalic vein at distal forearm. There was one mortality on fourth post operative day due to acute pulmonary edema. Two patients were lost to follow up. Of the remaining 33 patients, there was 3 immediate and 3 delayed failure and 27 patients $(81.81 \%$ ) had patent AVF at 6 months follow up including those who underwent renal transplant (Figure 1). 


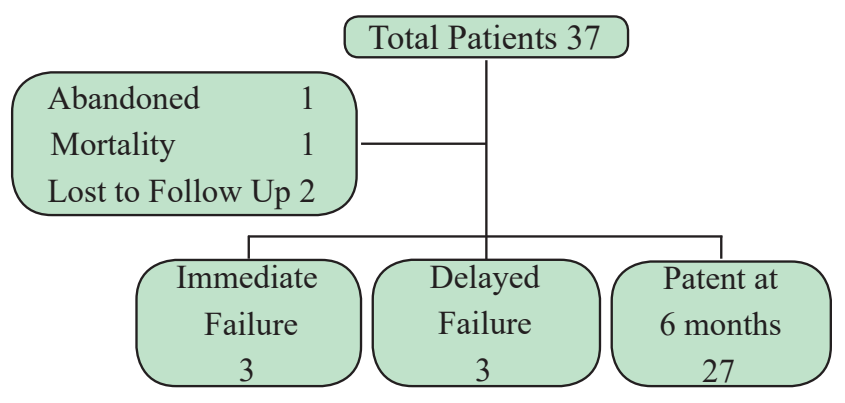

Figure 1: Consort diagram of Patient Flow and Follow Up

The maturation time varied between 3-7 weeks (mean +/- SD of $4.83+/-1.053$ ). Four patients (10.81\%) had complications in our study. Three patients had upper limb edema and one patient had hematoma. Four patients had repeat procedure in the vascular unit of other hospital, among them 3 had immediate failure and in one patient procedure was abandoned. Two patients (5\%) underwent transplant during the study period. There were no admissions in our study.

\section{Discussion}

The creation of AVF surgery is an important intervention for the outcome of patient on HD as it has a significant impact on survival. ${ }^{7}$ Our centre has a recently established Haemodialysis unit so we are getting more patients with renal failure and some of them require maintenance HD. The naïve AVF is the optimal vascular access for HD. ${ }^{8}$ All of the fistula created in our study was Radio cephalic (Brescia- Cimino) in left forearm though autologous radiocephalic AVF is the standard one in accordance with international recommendation. ${ }^{8}$ In a single center study from Sudan published in 2012, 78.1\% were distal fistulas. ${ }^{9}$ Similar result was published from Nigeria in which $90 \%$ of fistulas were distal fistulas. ${ }^{10}$

The mean age of patients in our study is 35.44 years which is almost ten years less than reported by other study. 9,11 In majority of patients $(n=17)$ the cause of renal failure was not identified when referred for AVF and among those diagnosed, the most common cause of renal failure was chronic glomerulonephritis $(n=9)$.

Time to first use of AVF is an important variable for clinical outcome. In our study 36 of 37 patients (97.29\%) were referred for vascular access after starting HD. This percentage is high than that reported from Australia, USA and Sudan (28\%, 78\% and $91.8 \%$ respectively)..$^{9,12,13}$ This may be due to requirement of emergency HD on first presentation or late referral to nephrologists.
Procedure was abandoned in one patient in our study due to inability to isolate a vein with good caliber in distal forearm and was referred to vascular unit of other centre. In $83.78 \%$ $(n=31)$ thrill or flow was noted in first post operative day which is quite similar to the results of study done in Sudanese patients with $87.7 \%$ immediate functioning fistula. ${ }^{9}$ The immediate failure rate in our study is $9.09 \%$ $(n=3)$ which varies from $10-30 \%$ in various studies. ${ }^{9,14,15}$ The failure rate in our series is $9.09 \%(n=3)$ at 6 months follow up which is lower than that reported from a study in Nigeria with $35.1 \%$ failure rate at 1 year. ${ }^{11}$ Twenty seven patients $(81.81 \%)$ out of 33 had a functioning fistula at 6 months follow up which was higher than that reported from Nigeria in 2013 with a 1 year patency rate of $63.2 \%{ }^{11}$

The maturation time in our study is 3 to 7 weeks (mean $+/-$ SD of $4.83+/-1.053)$ which is similar to that reported by other studies $\left(4.5+/-1.8,4.4+/-1.6\right.$ and 4.7+/- 1.8). ${ }^{9,11,16}$

The complications occurred in $10.81 \%(n=4)$ of patients in our study which is comparable to the study analyzing early follow up results that reported $11.4 \%$ complication rate. ${ }^{16}$ Three patients had arm edema out of which one patient had non functioning fistula since first post operative day and was referred to vascular unit of other centre and edema subsided on its own in other 2 patients. One patient developed haematoma on first post operative day which was evacuated in operation theatre but fistula was functional and was patent at 6months follow up. The common complication of AVF surgery is thrombosis. ${ }^{16} \mathrm{We}$ have not used ultrasound Doppler for our patients due to lack of logistics so we were not able to detect the possible thrombosis causing failure in our unsuccessful cases $(n=6)$.

Four patients (10.81\%) needed repeat surgery for creation of AVF in our study which is almost double than that reported from a single center study in Turkey with $5.98 \%$ patients requiring more than one surgery.16 Out of 4,1 case was abandoned and other 3 had immediate failure on clinical examination. All patients who needed repeat surgery were referred to vascular unit of other centre as there are limited sites for AVF creation, due to limited experience of performing surgeon and due to lack of vascular surgeon in same hospital if needed. None of the patients underwent interventions for immediate failure as we lack the facility and expertise for such secondary patency procedures.

All cases were done on day care basis. A study analyzing day care surgery for AVF creation reported an unscheduled admission rate of $6 \% .{ }^{17}$ The main reason being hemorrhage and malfunction almost accounting for $90 \%$ of cases. In our study 1 patient presented with acute pulmonary edema on 4 th day of creation of AVF and expired in emergency room. The cause of death may be he had missed two sessions of 
HD which resulted in acute pulmonary edema and may not be related with creation of AVF.

Only 5\% $(n=2)$ of patients had Renal transplantation during the 6 months follow up in our study which is almost half than stated by Turkish nephrology registry at $11 \%$ renal transplant rate among ESRD patients. ${ }^{18}$

Though it was a single surgeon study, the potential drawback of this study could be the chosen operative technique and exclusion of AVF made at other location. Other limitations include the lack of ultrasound Doppler for both pre and post operative assessment, small sample size and inclusion of cases whose cephalic vein was of good caliber and prominent on clinical examination and exclusion of the borderline ones.

The creation of AVF was included in the overall activity of the general surgical operative list. The ultimate outcome of fistula surgery is its use for HD. Our results regarding maturation time, 6 months patency rate with a less number of well known minor complications makes us believe that the AVF creation surgery for HD can be performed with no detrimental effect on patient care in a general surgical unit, it also reduces patient and party anxiety and stress of visiting other centre for surgery and can be incorporated in a overall general surgery activity.

\section{Conclusions}

The creation of AVF for HD performed by a general surgeon having vascular skill is a safe procedure in patients with good size vein and artery with satisfactory outcome and acceptable level of complications.

\section{References}

1. Mendelssohn DC, Ethier J, Elder SJ, Saran R, Port FK, Pisoni RL. Haemodialysis vascular access problems in Canada: results from the Dialysis Outcomes and Practice Patterns Study (DOPPS II). Nephrol Dial Transplant. 2006;21(3):721-8.

2. Dilege S, Baktiroglu S, Basar Y, Genc FA, Ozgur F. Basilic vein transposition as vascular access for hemodialysis. Turkish $\mathrm{J}$ Thorac Cardiovasc. 1995;3:140-142.

3. Pisoni RL, Young EW, Dykstra DM, etal. Vascular access use in Europe and the United States: results from the DOPPS. Kidney Int. 2002;61(1):305-316.

4. Dhingra RK, Young EW, Hulbert- Shearon TE, Leavey SF, Port FK. Type of vascular access and mortality in U.S. hemodialysis patients. Kidney Int. 2001;60(4):1443-1451.

5. McGrogan DG, Maxwell AP, Inston NG, Krishna $\mathrm{H}$, Field H. Preserving Arteriovenous fistula outcomes during surgical training. J Vasc Access. 2014;15(6):474-480.

6. Hirth RA, Turenne MN, Woods JD, Young EW, Port FK, Pualy MV. Predictors of type of vascular access in hemodialysis patients. JAMA. 1996;276(16):1303-8.

7. Allon M, Daurgidas J, Depner TA, Greene T, Ornt D, Schwab SJ. Effect of change in vascular access on patient mortality in hemodialysis patients. Am J Kidney Dis. 2006;47(3):469-477

8. Clinical practice guidelines for vascular access: update2000. Am J Kidney Dis. 2001;37(1 Suppl 1):137-81.

9. Ahmed GM, Mansor MO, Elfaith M, Khalid KE, Ahmed ME. Outcomes of arteriovenous fistula for hemodialysis in Sudanese patients: Single-Centre experience. Saudi Journal of Kidney Disease and transplantation. 2012;23(1):152-157.

10. Bakari AA, Nwankwo EA, Yahaya SJ, Mubi BM, Tahir BM. Initial five years of arteriovenous fistula creation for hemodialysis vascular access in Maiduguri, Nigeria. Int J Cardiovasc Res. 2007;4(2):1-6.

11. Alhassan SU, Adamu B, Abdu A, Aji SA. Outcomes and complications of permanent hemodialysis vascular access in Nigerians: A single centre experience. Ann Of Afr Med. 2013;12(2):127-130.

12. Di lorio BR, Bellizzi V, Cilio N etal. Vascular access for haemodialysis: the impact on morbidity and mortality. J Nephrol. 2004;17(1):19-25

13. Fitzgerald JT, Schanzer A, Chin AL, McVicar JP, Perez RV, Troppmann C. Outcomes of upper arm arteriovenous fistulas for maintenance hemodialysis access. Arch Surg. 2004;139(2):201-8

14. Rooijens PP, TOrdoir JH, Stijnen T, Burgmans JP. Radiocephalic wrist arteriovenous fistula for hemodialysis: Metaanalysis indicates a high primary failure rate. Eur J Vasc Endovasc Surg. 2004;28:583-9

15. Malovrh M. Native arteriovenous fistula: pre-operative evaluation. Am J Kidney Dis. 2002;39(6):1218-25.

16. Iyem H. Early follow up results of arteriovenous fistulae created for hemodialysis. Vascular Health and Risk Management. 2011;7;321-325.

17. Almonacid PJ etal. Ambulatory surgery of patients with arteriovenous fistulas for hemodialysis. Intregrated activity in a department of general surgery. Nefrologia. 2010;30(4):452-7

18. Turkish society of Nephrology. Registry of the Nephrology, Dialysis and Transplantation in Turkey. Registry 2004. Omega CRO. Istanbul, Turkey: Turkish Society of Nephrology;2005. 